\title{
Controlling molecular self-assembly: from amyloid oligomerization and therapy to novel biomaterials and technological applications in nanomedicine
}

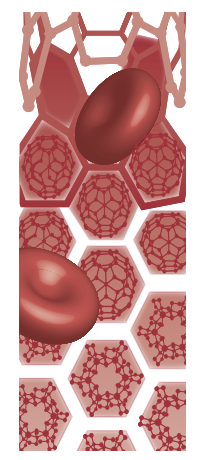

\begin{abstract}
"We believe that a better understanding of supramolecular associations could lead to improved understanding and control of biological processes for new medical applications."
\end{abstract}

Keywords: amyloid diseases $\bullet$ drug development $\bullet$ material science $\bullet$ molecular recognition - nanomaterials • self-assembly

The control of molecular self-assembly at the nanoscale is a novel and promising direction for nanomedicine since many physiological and pathological processes require interactions between biomolecular building blocks to form ordered nanoassemblies. The control of the controlled self-association of proteins, polypeptides and even simple metabolites to form ordered amyloidal nanofibrils and nanoscale oligomers is of great significance. This path is directly related to a major branch of nanoscience and nanotechnology that is involved with the design and bottom-up fabrication of nanostructures of unique morphologies and physicochemical properties by the process of molecular selfassembly. The association of small building blocks, via an array of specific and noncovalent interactions, can lead to the formation of various structures with complex architectural characteristics and functional features. The molecular manipulation of such assemblies at the nanoscale is a very promising direction for numerous technological applications, and is also related to the important field of supramolecular polymers [1]. We believe that a better understanding of supramolecular associations could lead to improved understanding and control of biological processes for new medical applications.

The biological world is a prime example of supramolecular polymers and copolymer assemblies, and thus a thorough understanding of molecular self-assembly could enable the development of novel therapeutic agents. Nature in general is characterized by the formation of complex assemblies using simple building blocks that self-associate to form functional noncovalent entities. Biology is an important source for the development of the field of organic nanotechnology since living biological systems are in fact comprised of nanodevices and nanomachines. Living systems allow the formation of functional ordered coassemblies such as pumps, motors and sensors from elementary building blocks through self-organization processes with no blueprint, fabrication or miniaturization processes. In some cases, the formation of ordered supramolecular structures is not related to physiological properties but rather to pathological properties. A key example of the enormous medical importance is the formation of $7-10-\mathrm{nm}$ amyloid fibrils that are related to many human disorders, including Alzheimer's diseases, Parkinson's disease and Type 2 diabetes [2]. The amyloid structures are well ordered and in addition to their nanometric scale, they also possess features such as a typical $\mathrm{x}$-ray fiber diffraction pattern and birefringence upon binding to fluorescent dyes. The very efficient formation of ordered amyloid nanofibrils from quite simple building blocks provides an oppor-

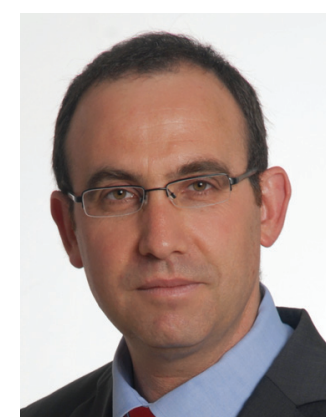

Ehud Gazit

Department of Molecular Microbiology \& Biotechnology, George S. Wise Faculty of Life Sciences, Department of Materials Science \& Engineering, lby \& Aladar Fleischman Faculty of Engineering, Tel Aviv University, Tel Aviv 69978, Israel

Tel.: +97236409030 ehudg@post.tau.ac.il 
tunity to further our understanding of molecular selfassembly on the one hand and to develop new tools to control self-assembly - namely, the development of therapeutic agents - on the other hand. Besides their role in pathological processes, amyloid fibrils seem to be central assembly modules in physiological process such as the formation of biofilm and hyphae by several microorganisms [2]. Moreover, amyloid can serve as a catalytic entity. The remarkable ability of protein and peptide sequences to readily form amyloid-like assemblies might have had a role in the very early stages of life since amyloid-like structures could provide both close-caged environments as well as catalytic entities to allow chemical evolution that predated the relatively complex RNA oligomer structures [3,4].

Attempts to understand the minimal requirements needed to form well-ordered amyloids [5] and the pursuit of minimalistic approaches led to the identification of a very simple dipeptide motif, which is derived from $A \beta$ polypeptides that form amyloid fibrils in Alzheimer's disease [6]. Several studies demonstrated that very short peptide fragments (e.g., tetrapeptides) can form canonical amyloid structures that have all the features described above. Moreover, the diphenylalanine motif, from the core recognition module of the $A \beta$, can readily form ordered structures that share spectroscopic signatures and some structural properties with amyloid fibrils. It is assumed that the remarkable ability of this dipeptide to direct the formation of highly ordered supramolecular structures is related to the high propensity of this aromatic pair to direct the formation of ordered domains. These structures readily form nanotubular structures and its analogs can form other architectures at the nanoscale. Interestingly, it was recently discovered that the formation of nanostructures by this dipeptide is accompanied by the generation of reactive oxygen species, which is similar to observations reported during the formation of amyloid fibrils from full-length amyloidogenic polypeptides [7]. Another interesting observation that may shed light on the physiological relevance of the diphenylalanine motif is the identification of an E. coli enzyme that specifically targets the Phe-Phe bond [8]. The development of a specific cellular mechanism to avoid the presence of such exposed Phe-Phe motifs may imply the high tendency of this module to associate into ordered assemblies that may have a cytotoxic effects - as observed with amyloid assemblies [8]. While Phe-Phe motifs are usually buried in the hydrophobic cores of proteins in multicellular organisms, in bacteria dramatic changes in environmental parameters such as temperature or ionic strength can lead to the unfolding of proteins and the exposure of the diphenylalanine motif with a high tendency to associate into ordered supramolecular assemblies. A virtual screen of all 400 naturally occurring dipeptides indicated the specific propensity of aromatic dipeptides to form aggregative structures [9]. It should be noted that the propensity of aromatic dipeptides is much higher than aliphatic dipeptides, which do not show a significant aggregation propensity [9]. More recent studies have demonstrated that even simpler structures, such as the phenylalanine amino acid in phenylketonuria, may form amyloid-like nanostructures that are most likely related to amyloidal pathologies [10]. Thus, self-assembly in general and amyloidal association in particular may serve as a very promising drug target that requires further exploration.

The peptide assemblies that are formed are not only well ordered, but they also have unique physicochemical properties [11-13]. This includes semiconductive, piezoelectric and optical properties. In fact, the observed optical properties of the dipeptide assemblies are similar to those found in amyloid structures made from much larger proteins and polypeptides [14]. These common characteristics between nanoassemblies (such as nanotubes and nanospheres) made of dipeptides and protein- and polypeptide-based amyloid assemblies, once again emphasize the interplay between nanomaterials and nanomedicine, and the relevance of the formed dipeptide structures as to the physiological and pathological amyloid states. The observed optical signature of the amyloid and dipeptide assemblies could also be relevant for the development of diagnostic tools to monitor the formation of amyloid fibrils and their inhibition. The dipeptide nanostructures also have a Young's modulus of up to $30 \mathrm{GPa}$ for the nanotubes and $275 \mathrm{GPa}$ for the nanosphere. The piezoelectric properties of the nanostructures are comparable to the best inorganic piezoelectric materials, such as lithium niobate. The application of nanostructures was demonstrated in various fields including electrochemical biosensors, tissue engineering, drug delivery and molecular imaging [15]. Among other things, the ability of diphenylalanine to form hollow nanotubes with remarkable persistence lengths that could be biologically proteolyzed was utilized in its application as a degradable casting mold for the fabrication of metallic nanowires [6]. Inkjet technology and vapor deposition were used to coat surfaces and from peptide 'nanoforests' in a highly controlled manner. Finally, the assembly of peptides was used as a driving force to propel nanoboats in the conversion of the energy of assembly into kinetic energy [16].

Nanotechnology and nanoscience provides new paradigms to understand molecular self-assembly related to neurodegenerative and other amyloid disorders [17-20]. The newly identified recognition mod- 
ules allow the design of novel therapeutic agents that inhibit amyloid oligomerization and affect structural transitions within the proteins and polypeptides, for example amyloid formation inhibitors. Control of the assembly interaction by designing peptides and small molecules that bind to the recognition interfaces of the amyloid-forming modules and thus block further growth is highly promising. There are several ways to approach this challenge. One approach includes high-throughput screening and rationalization of the molecular basis of inhibition, while other approaches are based on rational design and iterative cycles of optimization to select efficient and safe chemical entities that can modulate the assembly process. Highresolution studies including solution nuclear magnetic resonance have provided direct experimental proof for the targeting of recognition site by inhibitory agents. In recent years, it has become quite evident that it is most likely not the mature fibrillar structures that cause the cytotoxic effects that are observed in amyloid diseases, but rather earlier transient assemblies. This again emphasizes the importance of understanding the early molecular recognition and self-assembly processes. The ability to modulate the process at the initial phase of association may provide a very important therapeutic avenue.

\section{References}

1 Aida T, Meijer EW, Stupp SI. Functional supramolecular polymers. Science 335, 813-817 (2012).

2 Cherny I, Gazit E. Amyloids: not only pathological agents but also ordered nanomaterials. Angew. Chem. Int. Ed. Engl. 47, 4062-4069 (2008).

3 Rufo CM, Moroz YS, Moroz OV et al. Short peptides self-assemble to produce catalytic amyloids. Nat. Chem. 6, 303-309 (2014).

4 Carny O, Gazit E. A model for the role of short self-assembled peptides in the very early stages of the origin of life. FASEB J. 19, 1051-1055 (2005).

5 Lu K, Guo L, Mehta AK et al. Macroscale assembly of peptide nanotubes. Chem. Commun. 2007 14, 2729-2731 (2007).

6 Reches M, Gazit E. Casting metal nanowires within discrete self-assembled peptide nanotubes. Science 300, 625-627 (2003).

7 Souza MI, Silva ER, Jaques YM, Ferreira FF, Fileti EE, Alves WA. The role of water and structure on the generation of reactive oxygen species in peptide/hypericin complexes. J. Pept. Sci. 20, 554-562 (2014).

8 Lütticke C, Hauske P, Lewandrowski U, Sickmann A, Kaiser M, Ehrmann M. E. coli LoiP (YggG), a metalloprotease hydrolyzing Phe-Phe bonds. Mol. Biosyst. 8, 1775-1782 (2012).
In summary, the amyloid system provides a unique platform to understand self-assembly at the nanoscale, to define minimal recognition modules and to explore physical and chemical properties at the nanoscale. The identification of recognition modules that promote self-assembly allows the fabrication of new nanomaterials of various geometries and architectures and their utilization in various applications. Finally, the ability to control self-assembly at the nanoscale will be vital in the development of therapeutic agents that target this process. Some of the principles that we understand from the manipulation of the relatively simple amyloid self-assembly could be utilized for the development of drugs that will target other self-assembly processes such as capsid assembly to provide new antiviral therapies.

\section{Financial \& competing interests disclosure}

The author has several licensed patents in the field of amyloid formation inhibition and nanomaterials. The author has no other relevant affiliations or financial involvement with any organization or entity with a financial interest in or financial conflict with the subject matter or materials discussed in the manuscript apart from those disclosed.

No writing assistance was utilized in the production of this manuscript.

9 Frederix PW, Ulijn RV, Hunt NT, Tuttle T. Virtual screening for dipeptide aggregation: toward predictive tools for peptide self-assembly. J. Phys. Chem. Lett. 2, 2380-2384 (2011).

10 Adler-Abramovich L, Vaks L, Carny O et al. Phenylalanine assembly into toxic fibrils suggests amyloid etiology in phenylketonuria. Nat. Chem. Biol. 8, 701-706 (2012).

11 Yan X, Zhu P, Li J. Self-assembly and application of diphenylalanine-based nanostructures. Chem. Soc. Rev. 39, 1877-1890 (2010).

12 Hauser CA, Zhang S. Nanotechnology: peptides as biological semiconductors. Nature 468, 516-517 (2010).

$13 \mathrm{Kim}$ JH, Ryu J, Park CB. Selective detection of neurotoxin by photoluminescent peptide nanotubes. Small 7(6), 718-722 (2011).

14 Pinotsi D, Buell AK, Dobson CM, Kaminski-Schierle GS, Kaminski CF. A label-free, quantitative assay of amyloid fibril growth based on intrinsic fluorescence. Chembiochem 14, 846-850 (2013).

15 Silva RF, Araújo DR, Silva ER, Ando RA, Alves WA. L-diphenylalanine microtubes as a potential drug-delivery system: characterization, release kinetics, and cytotoxicity. Langmuir 29, 10205-10212 (2013).

16 Ikezoe Y, Washino G, Uemura T, Kitagawa S, Matsui H. Autonomous motors of a metal-organic framework powered by reorganization of self-assembled peptides at interfaces. Nat. Mater. 11, 1081-1085 (2012). 
17 Porat Y, Abramowitz A, Gazit E. Inhibition of amyloid fibril formation by polyphenols: structural similarity and aromatic interactions as a common inhibition mechanism. Chem. Biol. Drug Des. 67, 27-37 (2006).

18 Kirschner DA, Gross AA, Hidalgo MM et al. Fiber diffraction as a screen for amyloid inhibitors. Curr. Alzheimer Res. 5, 288-307 (2008).
19 Lee JS, Ryu J, Park CB. High-throughput analysis of Alzheimer's beta-amyloid aggregation using a microfluidic selfassembly of monomers. Anal. Chem. 81, 2751-2759 (2009).

20 Li M, Xu C, Wu L, Ren J, Wang E, Qu X. Self-assembled peptide-polyoxometalate hybrid nanospheres: two in one enhances targeted inhibition of amyloid $\beta$-peptide aggregation associated with Alzheimer's disease. Small 9, 3455-3461 (2013). 